\title{
Characterization of the effect of microfracture surgery and angle of incidence on the structural properties of femoral bone
}

\author{
Li (Danny) Liang ${ }^{1}$, Alexis Wagner ${ }^{1}$, Justin Steeds ${ }^{1,2}$, Mark Hurtig $^{2}$, and Karen Gordon ${ }^{1}$ \\ ${ }^{1}$ School of Engineering, College of Physical and Engineering Science, University of Guelph, Guelph, ON Canada. \\ ${ }^{2}$ Comparative Orthopaedic Research Laboratory, Ontario Veterinary College, University of Guelph, Guelph ON Canada. \\ Faculty supervisor: Dr.Karen Gordon. \\ For correspondence, please email: Iliang@uoguelph.ca.
}

\begin{abstract}
Microfracture surgery is one of the most common treatment options for knee osteoarthritis, a chronic inflammatory disease of the knee joint. However, a recent study by Theodoropoulos et al. (2012) has shown that there are significant variations in the angle of surgical awl used by surgeons in microfracture surgery as well as in the rehabiliation regimen prescribed following surgery. The purpose of this experiment was to characterize the structural properties of femoral bone before and after microfracture, with varying awl angles of impact. Microfracture at three different awl angles of impact, each between $\mathbf{0}$ and $\mathbf{3 0}$ degrees, was conducted on bone cubes cut from femoral condyles. Uniaxial compressive testing was conducted pre- and post- microfracture to determine changes in the stiffness of the bone. The results from this study show that there are significant decreases in the stiffness of femoral bone following microfracture surgery. However, different awl angles do not seem to result in significant decreases in bone stiffness. In addition, these results suggest that at the relatively low awl angles that were tested, differences in the angle of surgical awl used do not significantly affect the structural integrity of knee bone. Uniaxial testing appears a useful tool for characterizing the structural properties of bone, and further animal studies on the stiffness of bone at different timepoints following microfracture surgery could assist physicians in prescribing more suitable rehabilitation regimens.
\end{abstract}

Keywords: knee osteoarthritis; microfracture; biomechanical testing; femoral condyle; awl angle; bone stiffness

\section{Introduction}

Osteoarthritis (OA) is a chronic disease with progressive, debilitating effects that affects more than one in eight Canadians (Bombardier et al. 2011). Within the next 30 years, it is predicted that more than one in four Canadians will be living with $\mathrm{OA}$, with a higher incidence among females, athletes, and adults over 60 (Zhang and Jordan 2010). OA of the knee results from biomechanical stress on the articular cartilage and subchondral bone and causes symptoms that include pain, morning stiffness, swelling, and functional impotence of the affected joint. Since cartilage has a limited ability to self-repair and regenerate, a treatment with $100 \%$ efficacy does not exist for OA. With such a high prevalence and no assured cure, $\mathrm{OA}$ is a significant economic burden on the Canadian healthcare system.

\section{Treatment options}

Focal cartilage defects are widely associated with the development of OA (Magnussen et al. 2008). Thus, treatment options involve various methods that aim to repair cartilage defects by encouraging regeneration and thickening of the articular cartilage. Non-surgical treatments including diet, rehabilitation, weight loss and intraarticular injections generally only provide pain relief and are not a permanent solution (Smith et al. 2005). Common surgical treatments for repairing full-thickness cartilage defects include microfracture, mosaicplasty, autologous chondrocyte implantation and total joint replacement (Gobbi 2010). Microfracture is the primary treatment for focal cartilage defects due to its simplicity, low cost, and less invasiveness than would be provided by other techniques.

The microfracture technique, developed by Richard Steadman in the early 1980s, generates fibrocartilage in areas of full-thickness chondral lesions (Steadman et al. 2010). An offset awl is hammered into the debrided articular surface to penetrate through the subchondral bone plate (SBP). Once the SBP is breached, the underlying bone marrow bleeds out onto the defect. Marrow-based progenitor cells and growth factors form a blood clot on the defect. The stem cells differentiate into fibroblasts and undergo further hyalinization and chondrification to become a cartilaginous tissue. Microfracture has shown strong efficacy in reducing 
pain and swelling and improving function. It is also costeffective and with few complications (Mithoefer et al. 2005).

Steadman's widely-adopted microfracture protocol recommends debriding full thickness lesions down to the subchondral bone plate, thereby creating a clean square around the defect. Debriding must not disrupt the structural integrity of the SBP (Steadman et al. 1997). Microfracture holes are created 3-4 mm apart so that the holes do not break into each other (Steadman et al. 1997). The depth of the microfracture holes must be $2-4 \mathrm{~mm}$ deep so that fat droplets emanate from the marrow cavity. The effectiveness of microfracture depends primarily on the surgical technique, the age of the patient, the size of the lesion, and the rehabilitation program (Hannon et al. 2013).

Despite Steadman's detailed guidelines, there is still a great deal of variation in the microfracture surgical technique. A 2012 study revealed that Canadian orthopedic surgeons vary greatly in their surgical techniques and postoperative rehabilitation programs (Theodoropoulos et al. 2012). Theodoropoulos et al. observed a large variance in prescribed post-operative weight bearing status and in the duration of altered weight bearing status, which ranged from 2-12 weeks. Additionally, $62 \%$ of surgeons claimed that the awl tip had a tendency to slip when creating holes in the SBP. This slippage was reported to sometimes cause an increase in the angle of the awl pick. When the long axis of a microfracture hole tilts, a stress concentration around the lip of the hole may develop, thus increasing the likelihood of fracture failure around the microfracture hole (Theodoropoulos et al. 2012).

Limited research exists to evaluate the effect of incident awl angle on the resultant mechanical strength of the bone. The purpose of this investigation was to determine the effects of varying microfracture treatments on the mechanical properties of knee bone. The study's aim was to provide insight on the effect of microfracture holes on the acute structural integrity of the subchondral bone, and assess if microfractured holes with a high angle of awl impact increase susceptibility to fracture failure around the hole site.

\section{Materials and Methods}

\section{Specimen preparation}

Lateral femoral condyles were obtained from human cadaveric knees and stored at $-20^{\circ} \mathrm{C}$ until use. Subchondral bone cubes of $15 \mathrm{~mm} \times 15 \mathrm{~mm} \times 10 \mathrm{~mm}$ were cut from the condyles on a slow speed diamond saw (Model No. 800-900, LECO Corp. St. Joseph, MI, USA) using parallel diamond saw blades (M4D220-N100M99-1/8, Worcester, MA, USA) (Figure 1). Anterior (AL) and posterior (PL) samples were cut from each condyle. The bone samples and blades were maintained under continuous irrigation with saline solution during the cutting process. After cutting, the cartilage was debrided using a scalpel and dental scraper. Bone cube dimensions were recorded using digital calipers. In total, 38 bone cubes were prepared: there were 12 bone cubes in the 0 degree group, 11 bone cubes in the 15 degree group and 10 bone cubes in the 30 degree group.

Aluminum bone mounting blocks were custom-machined from $64 \times 64 \times 25.4 \mathrm{~mm}$ solid aluminum blocks with a square opening in the top-middle for bone-cube mounting. The custom mounts were used as the lower plate for compressive testing (Figure 2). Dental acrylic was placed into the opening (Lang Dental Mfg Co., Inc, Wheeling, IL), and the bone cubes were then tightly wrapped in plastic film, lightly coated with Vaseline, and pressed into dental acrylic to cure.

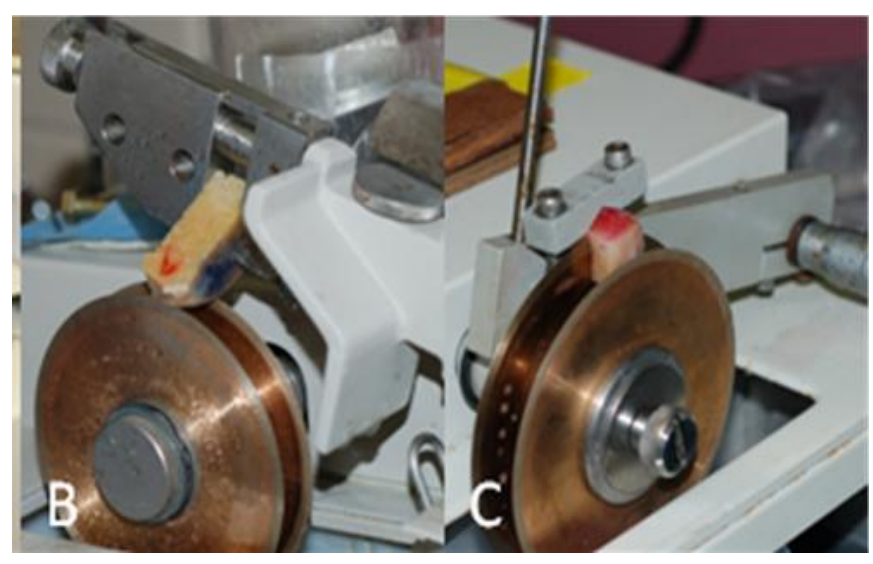

Figure 1. A diamond saw cutting of $15 \times 15 \times 10 \mathrm{~mm}$ bone cubes.

\section{Microfracture procedure}

A template and pen were used to indicate the microfracture sites on the articular surface of each sample. The holes were separated by a distance of $3 \mathrm{~mm}$, as recommended by Steadman. A $3 \mathrm{~mm}$ distance was also maintained from the edges of the bone cube. Microfracture holes were made using a 20 degree Arthrex awl (Arthrex, Inc., Naples FL, USA). The appropriate bone cube sample was placed into the cured dental cement mold within the aluminum block to secure the sample during the procedure. Three angles of awl penetration were tested: 0 degrees, 15 degrees, and 30 degrees. The awl angles of impact were determined using a digital angle finder tool, which was secured to the shaft of the Arthrex awl using the internal magnet. Since the Arthrex awl already had a 20 degree angle on it, the penetration angle of the pick had to be adjusted accordingly. Thus, 0 degrees, 15 degrees, and 30 degrees were displayed as 70 degrees, 55 degrees and 40 degrees respectively on the digital angle finder. A metal mallet was used to hammer the awl into the cube until the $3 \mathrm{~mm}$ gold tip on the pick of the awl was fully indented into the subchondral bone. The same procedure was used for all hole sites, and all holes weer made in the posterolateral direction.

\section{Mach1 biomechanical testing}

Non-destructive uniaxial compressive testing was performed using a Mach-1 Micromechanical Testing System (Biomomentum Inc., Laval, QC, H7L 5C1). Three replicates were done using each sample. The aluminum custom blocks 


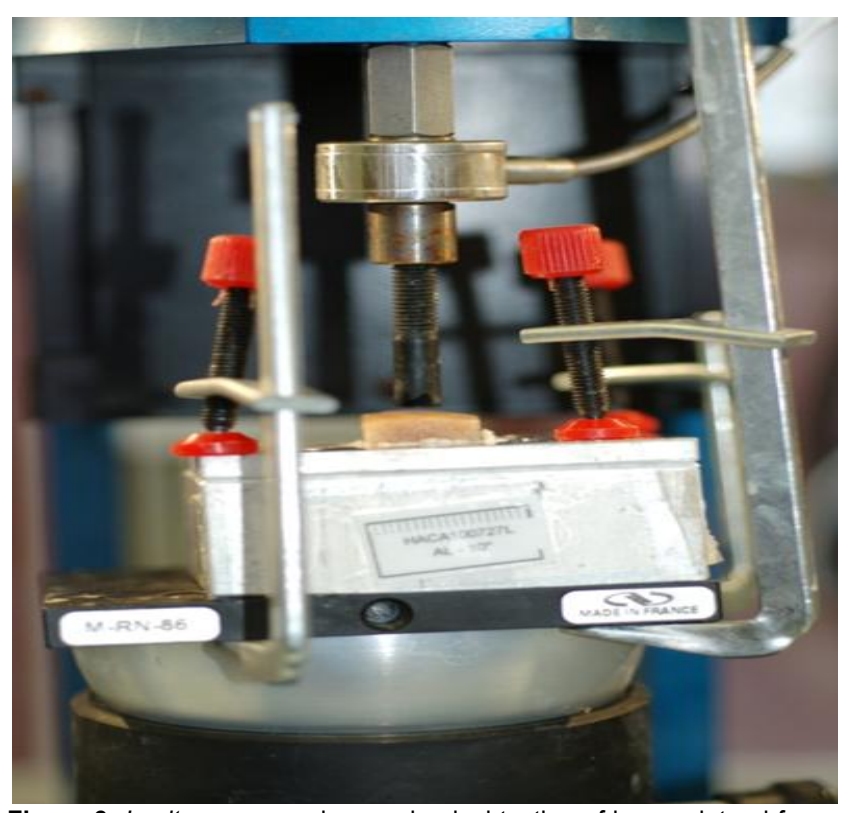

Figure 2. In vitro compressive mechanical testing of human lateral femoral condyle subchondral bone plate using a Mach 1 Micromechanical Testing System (Biomomentum Inc., Laval, QC, Canada).

were secured to a rotational stage using clamps, as shown in Figure 2. The sample was centered about the upper test platen $(6.3 \mathrm{~mm}$ diameter), orienting the lower $x-y$ table and rotational stage so that the tangent to the curve of the articular surface was approximately parallel with the face of the upper platen. A thin film of mineral oil was applied to the face of the upper platen with a cotton swab to create a frictionless surface. The upper platen was manually lowered to approximately $1 \mathrm{~mm}$ from the bone surface in order to avoid damaging the test specimen. A test sequence was written using the Mach-1 software and a "find contact" routine was used in load control to lower the platen at a velocity of $3 \mu \mathrm{m} / \mathrm{s}$ until a load of $2 \mathrm{~g}$ was reached. After contact with the surface was made, a $0.25 \%$ pre-strain at a strain rate of $0.01 \mathrm{~s}^{-1}$ (move relative) was applied, followed by a dynamic sinusoid load of amplitude of $0.05 \%$ strain and a frequency of $1 \mathrm{~Hz}$ for 20 cycles. Accordingly, samples were compressed to an upper strain of $0.3 \%$ to remain consistent with in vivo studies conducted on several species that showed peak strain on bone surfaces of approximately $0.3 \%$ (Burr et al. 1996, Nobel et al. 2003, Rubin et al. 1985)

\section{Statistical analysis}

Three replicates of non-destructive testing were performed before and after microfracture on each test sample. A load-displacement curve was plotted for each test replicate using Microsoft Excel software. The stiffness was calculated from the slope of the linear portion of the curve with a coefficient of determination $\left(r^{2} \geq 0.95\right)$ and averaged from the three test replicates. After all of the raw data were compiled, t-tests (at 95\% confidence) and the graphing of results were performed using Microsoft Excel software.

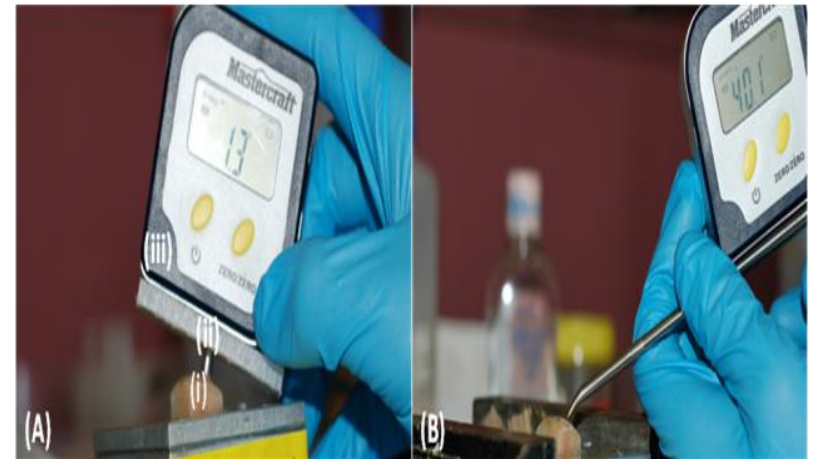

Figure 3. (A) Taring to calibrate the digital angle measurement tool (iii) using the custom tool (ii) to find the tangent to the articular surface about each hole site on the bone cube sample (i). (B) Arthrex awl at a 30 degree angle orientation to tangent of subchondral bone plate surface.

\section{Results and Discussion}

As expected, there were noticeable decreases in bone stifness in all three angle groups following the microfracture procedure. Statistically significant differences were found in the 0 and 15 degree groups but not in the 30 degree group ( 0 degrees: $p=0.013 ; 15$ degrees: $p=0.018$; 30 degrees: $\mathrm{p}=0.13$ ). Results highlighted in Figure 4 demostrate that uni-axial compressive testing may serve as an useful tool in determining the effects of surgical procedures, such as microfracture, on the structural properties of bone. The use of this method in future animal studies on the stiffness of bone at different timepoints following microfracture surgery could help physicians better understand the process of bone formation following microfracture. Eventually, this could lead to the prescription of and more suitable rehabiliation regimens, thereby decreasing the large variations in rehabilitation regimens currently being prescribed.

When comparing the decreases in stiffness between the three angle groups, no statistical differences were determined ( 0 and 15 degrees: $\mathrm{p}=0.85 ; 0$ and 30 degrees: $0.76 ; 15$ and 30 degrees: $p=0.65)$. Results highlighted in Figure 5 suggest that at the angles of awl impact tested in this experiment, which were between 0 degrees and 30 degrees, variations in the awl angle at the time of the surgery did not appear to significantly affect the structural properties of the underlying subchondral bone. Further research to examine the effects of higher awl angles on the structural properties of the subchondral bone may demonstrate more significant decreases in structural properties after microfracture. 


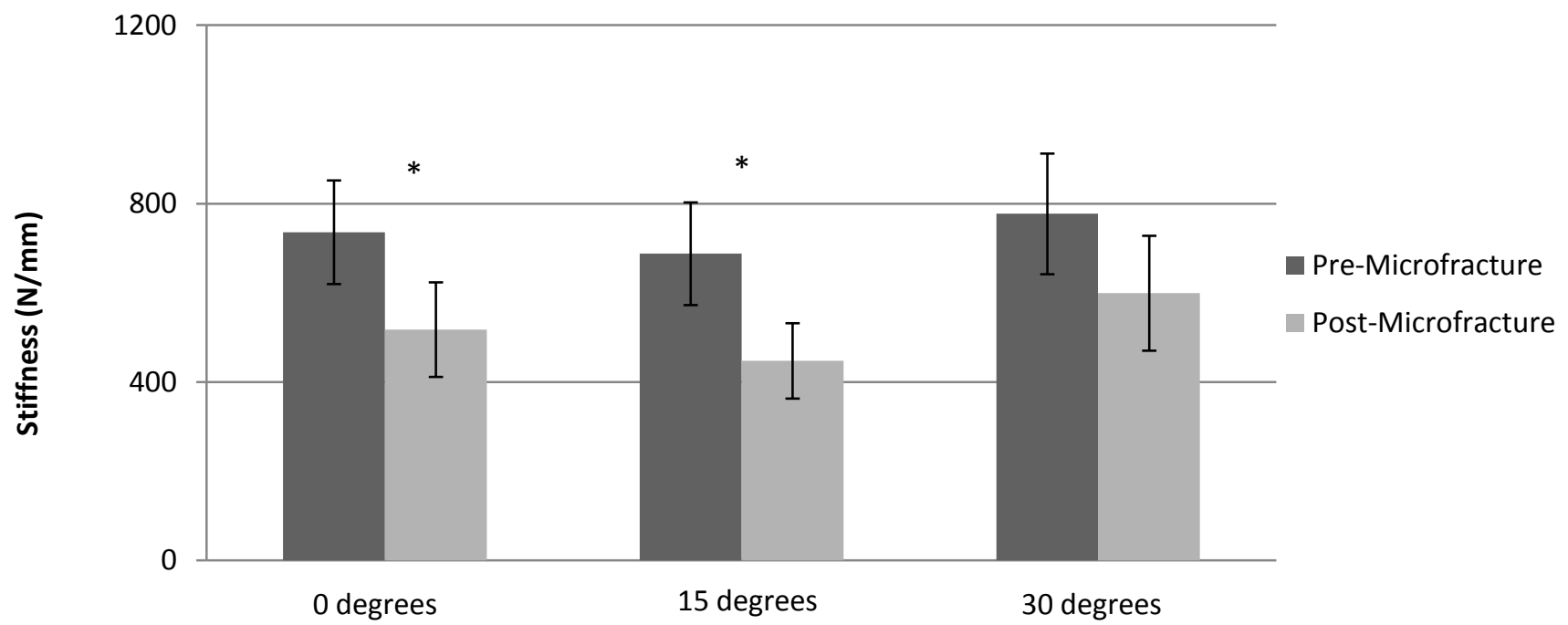

Awl Angle of Incidence

Figure 4. Bone stiffness before and after microfracture surgery at difference angles of incidence. Significant differences $\left(^{*}\right)$ in bone stifness were found between pre- and post-microfracture surgery groups at awl angles of 0 and 15 degrees ( 0 degrees: $p=0.013 ; 15$ degrees: $p=0.018$ ). No significant difference in bone stiffness between pre- and post-microfracture srugery groups were found at an awl angle of 30 degrees $(p=0.13)$.

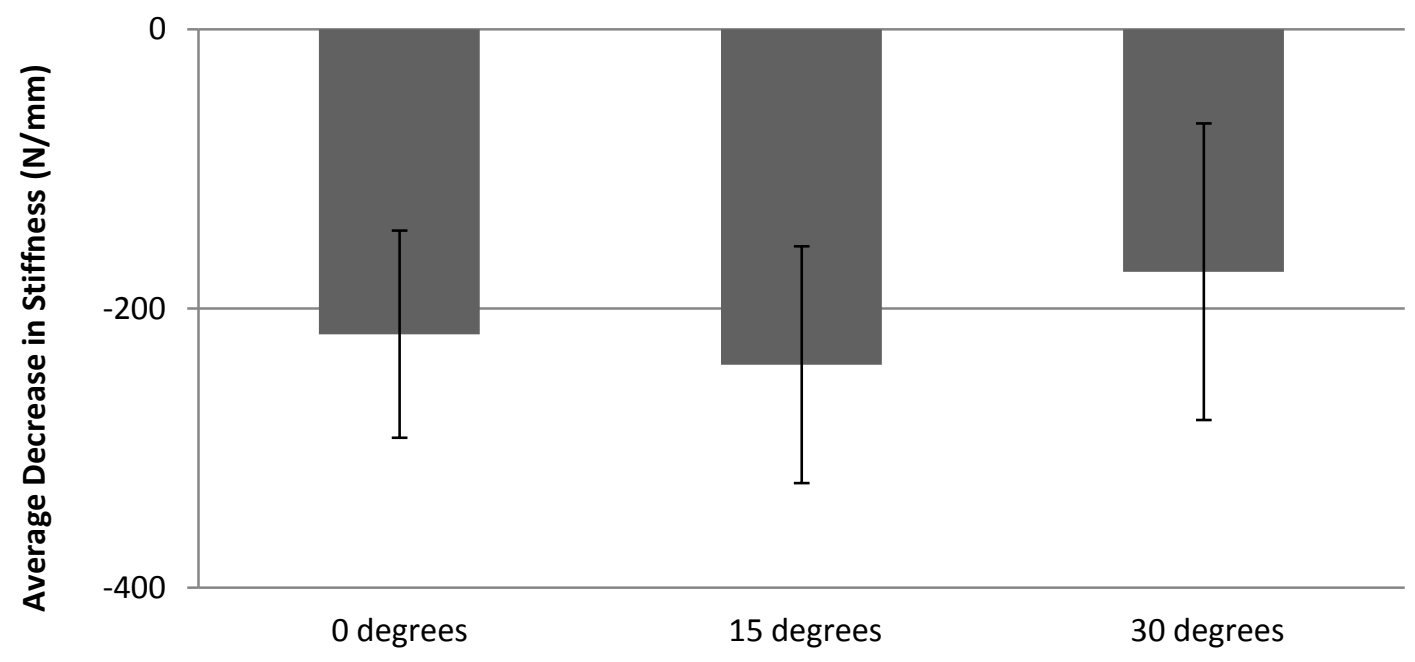

Awl Angle of Incidence

Figure 5. Awl angle of incidence versus average decrease in stifness $(\mathrm{N} / \mathrm{mm})$. No significant differences in stiffness following microfracture surgery were found when comparing the different awl angles of incidence ( 0 and 15 degrees: $p=0.85 ; 0$ and 30 degrees: $p=0.76 ; 15$ and 30 degrees: $p=0.65$ ). 


\section{Limitations}

As with any experiment, several sources of error were noted during the experiment. First, there were large variations in the sizes and curvatures of the condyles. As a result, the articular surfaces making contact with the platen during the uniaxial compressive testing were not always flat. This was especially prominent when the condyles were smaller, as the reduced radii of curvature resulted in greater difficulty cutting two $10 \mathrm{mmx} 10 \mathrm{mmx} 15 \mathrm{~mm}$ condyles that had flat articular surfaces. The use of more uniformly-sized condyles, for example by using condyles from cadavers of similar heights and weights, would minimize such variation.

Second, there was often significant slippage of the awl as a result of the the moment at the awl tip that was created by the hammering in of the awl with the mallet. Due to the far greater cross-sectional area of the mallet compared to that of the tip of the awl, it was challenging to ensure that the center of mass of the mallet struck the central axis of the awl each time. This slippage reduced the positional uniformity of the microfracture holes across different bone cubes due to deviations of the hole positions from that of the template. Slippage also caused significant deviations in the instantaneous awl angles from the indended angles. However, such awl slippages are likely comparable to those seen in clinics. The development of tools and methods to stabilize the awl during experiments and surgeries would be useful in alleviating this problem.

A third problem encountered was the imprecise positioning of the platen used for the compressive testing. This was due to the fact that the bone cubes, which were mounted onto custom aluminum blocks, were clamped onto a rotational stage that was integrated with the Mach1 Compressive Testing system. Since the rotational stage was aligned manually each time so that the platen was directly above the centre of the four microfracture holes, there were slight variations between samples in the accuracy of this manual alignment. In addition, any agitation during adjustments could have resulted in minor changes to the point of contact of the platen. In future experiments, this can be minimized with the use of a flat stage in place of the rotational stage.

Finally, since only 33 bone cubes were tested, a larger sample size would have been beneficial for gnerating more conclusive study results.

\section{Conclusion}

The results from this study show that there is a significant decrease in the stiffness of femoral bone following microfracture surgery. However, different awl angles of impact do not seem to result in significantly different decreases in the stiffness of the bone. These results suggest that uniaxial testing may be an useful tool for characterizing the structural properties of bone, and further animal studies of the stiffness of bone at different timepoints post-surgery could help physicians prescribe more suitable rehabilitation regimens. In addition, for the relatively low awl angles that were tested, differences in the angle of impact of the surgical awl do not appear to significantly affect the structural integrity of the knee bone. Further improvements in experimental design to minimize the aforementioned sourcesof error and the use of larger sample sizes would be useful in determining more conclusive results.

\section{Acknowledgements}

We wish to acknowledge the financial support of the Canadian Institutes for Health Research and the Canadian Arthritis Network. 


\section{References}

Bombardier C, Hawker G, Mosher D. 2011. The impact of arthritis in Canada: Today and over the next 30 years. Available from: http://www.arthritisalliance.ca/docs/20111022_2200_i mpact_of_arthritis.pdf

Burr, D. B., et al. 1996 "In vivo measurement of human tibial strains during vigorous activity." Bone 18(5) : 405-410.

Gobbi A. 2010. New possibilities for cartilage repair. European Musculoskeletal Review. 5(2): 64.

Hannon CP, Murawski CD, Fansa AM, Smyth NA, Do H, Kennedy JG. 2013. Microfracture for osteochondral lesions of the talus: A systematic review of reporting of outcome data. American Journal of Sports Medicine. 41(3): 689-695.

Magnussen RA, Dunn WR, Carey JL, Spindler KP. 2008. Clinical Orthopaedics and Related Research. 466(4): 952-962.

Mithoefer K, Williams RJ, Warren RF, Potter HG, Spock CR, Jones EC, Wickiewicz TL, Marx RG. 2005. The microfracture technique for the treatment of articular cartilage lesions in the knee. A prospective cohort study. Journal of Joint and Bone Surgery: American Volume. 87(9): 1911-1920.
Noble BS., et al. 2003 "Mechanical loading: biphasic osteocyte survival and targeting of osteoclasts for bone destruction in rat cortical bone." American Journal of Physiology-Cell Physiology 284(4) C934-C943.

Rubin, Clinton T., and Lance E. Lanyon. "Regulation of bone mass by mechanical strain magnitude." Calcified tissue international 37(4): 411-417.

Smith GD, Knutsen G, Richardson JB. 2005. A clinical review of cartilage repair techniques. Journal of Joint and Bone Surgery: British Volume. 87(4): 445-449.

Steadman J, Rodkey W, Briggs K. 2010. Microfracture: Its history and experience of the developing surgeon. Cartilage. 1(2): 78-86.

Steadman JR, Rodkey WG, Singleton SB, Briggs KK. 1997. Microfracture technique forfull-thickness chondral defects: Technique and clinical results. Operative Techniques in Orthopaedics. 7(4): 300-304.

Theodoropoulos J, Dwyer T, Whelan D, Marks P, Hurtig M, Sharma P. 2012. Microfracture for knee chondral defects: A survey of surgical practice among canadian orthopedic surgeons. Knee Surgery, Sports Traumatology, Arthroscopy. 20(12): 2430-2437.

Zhang Y, Jordan JM. 2010. Epidemiology of osteoarthritis. Clinics in Geriatric Medicine. 26(3): 355-336. 